\title{
RELAÇÃO ENTRE PESO AO NASCER, SEXO DO RECÉM-NASCIDO E TIPO DE PARTO
}

Arnaldo Augusto Franco de Siqueira * Florita Brickmann Areno **

Pedro Augusto Marcondes de Almeida*

Ana Cristina d'Andretta Tanaka *

SIQUEIRA, A.A.F. de et al. Relação entre peso ao nascer, sexo do recém-nascido e tipo de parto. Rev. Saúde públ., S. Paulo, 15:283-90, 1981.

RESUMO: Foram estudadas duas maternidades que atendem a população de níveis sócio-econômicos heterogêneos para avaliar a influência do peso do recém-nascido no tipo de parto. Foram analisados 16.095 nascimento. Verificou-se aumento da incidência de cesareanas com o aumento do peso do recém-nascido, nas duas maternidades, mas na maternidade particular a incidência foi mais elevada em relação à maternidade assistencial. Chamou a atenção o rato da não associação entre o tipo de parto e o peso do recém-nascido nas mulheres que tiveram atendimento particular. A predominância cio sexo feminino no grupo de peso de $2.500 \mathrm{~g}$ e menos foi estatisticamente significante e do mesmo modo a maior proporção do sexo masculino nos recém-nascidos com mais de $4.000 \mathrm{~g}$.

UNITERMOS: Peso ao nascer. Parto, assistência.

\section{INTRODUCAOO}

É clássico, segundo vários autores $2,6,7$ ao se estudar crescimento, o fato de que o peso e altura de recém-nascidos de termo do sexo masculino são maiores do que os do sexo feminino e este fenômeno ocorre durante todo o periodo de crescimento. Porém, são poucos os trabalhos que analisam o comportamento do peso de acordo com o sexo do recém-nascido. Entre estes, o de Yerushalmy e col.9, realizado em 1959-1961, na cidade de Nova York, que afirma ser a incidência de baixo peso ao nascer $(1.617 \mathrm{~g}-2.500 \mathrm{~g})$ maior no sexo feminino, com significância estatística, tanto para brancos como para não brancos: o de Abramovicz e Kass ${ }^{1}$ que afirma que uma das características do baixo peso é a maior incidencia de mulheres; e o de Cooke e Levine 4 , afirma que este fenômeno se deve ao menor ganho de peso das mulheres no último trimestre da gravidez.

Sabe-se também que existe variação do peso de acordo com a classe social ${ }^{3}$, porém não se sabe até que ponto os fatores sociais e a desnutrição influem nos valores do peso do recém-nascido.

Quanto aos recém-nascidos grandes para a idade gestacional, os fatores gené-

* Do Departamento de Saúde Materno-Infantil da Faculdade de Saúde Pública da USP - Av. Dr. Arnaldo, 715 - 01255 - São Paulo, SP - Brasil.

* Do Centro de Saúde "Geraldo de Paula Souza" da Faculdade de Saúde Pública da USP Av. Dr. Arnaldo, 715 - 01255 - São Paulo, SP - Brasil. 
SIQUEIRA, A.A.F. de et al. Relação entre peso ao nascer, sexo do recém-nascido e tipo de parto. Rev. Saúde públ., S. Paulo, 15:283-90, 1981.

tícos e os ambientais têm relação com o peso e, entre os ambientais, o nivel sócio-econômico elevado apresenta importância considerável.

Em relação ao tipo de parto, paralelamente ao progresso científico, vem ocorrendo um aumento universal na incidência de partos operatórios. No nosso meio o fenômeno não é diferente e, segundo alguns autores, este aumento é bastante evidente na clínica particular e nos hospitais que mantêm convênios particulares com a previdência social. O estudo realizado em 1968 por Gentile de Mello ${ }^{5}$ no sentido de avaliar a eficiência de determinado serviço em comparação com outros semelhantes. através da análise do tipo de parto em maternidades do Rio de Janeiro, revelou uma porcentagem de partos operatórios superior à média internacional em hospitais particulares e de convênio. Mostrou também a influência do fator econômico na escolha do tipo de parto, com a escolha da data para u nascimento do recém-nascido e concomitância da esterilização cirúrgica (laqueadura). Assim, a incidência de cesáreas girou em torno de $7 \%$ nos hospitais assistenciais, semelhante à dos Estados Unidos no mesmo ano; em três hospitais do Instituto Nacional de Assistência Médica e Previdência Social (INAMPS) variou de $9,7 \%$ a $14 \%$, nas maternidades contratadas pelo INAMPS variou de $4,6 \%$ a $31 \%$ e no regime de livre escolha houve variação de $15,6 \%$ a $51,6 \%$.

No entanto, devemos pensar na possibilidade do nível sócio-econômico afetar o peso do recém-nascido e situar o problema no sentido de até que ponto o peso do recém-nascido de família de nível sócio-econômico mais elevado é maior do que o daquele de classe baixa, necessitando-se, portanto, de maior número de partos operatórios decorrentes de desproporção céfalo pélvica.

Resolvemos estudar a influência do peso ao nascer sobre o tipo de parto, numa entidade que assiste a uma população de nivel sócio-econômico homogeneamente padronizado, comparando os resultados com os de uma maternidade particular.

\section{MATERIAL E METODOS}

Para levantamento dos dados foram verificados os livros de registro de nascimentos do berçário de uma maternidade assistencial e de uma maternidade particular, localizadas no município de São Paulo, em estudo referentes aos anos de 1974 e 1973, respectivamente.

Foram considerados todos os recém-nascidos vivos que deram entrada nos berçários das entidades que possuissem registros de peso, do tipo de parto e de sexo; foram desprezados aqueles que não apresentaram uma das variáveis em estudo.

Os recém-nascidos foram subdivididos em quatro grupos de acordo com o peso: 2.500 $\mathrm{g}$ e menos, de 2.501 a $3.000 \mathrm{~g}$, de $3.001 \mathrm{a}$ $4.000 \mathrm{~g}$ e acima de $4.000 \mathrm{~g}$. Cada grupo foi distribuido de acordo com o sexo e com o tipo de parto. Optou-se pelo ano de 1974 na maternidade assistencial porque foi o último ano em que os registros apresentavam uma única via de entrada.

Os resultados foram comparados com os de um época próxima no segundo hospital e tabulados.

\section{RESULTADOS E DISCUSSAO}

$\mathrm{Na}$ maternidade assistencial deram entrada no berçário 12.919 recém-nascidos vivos; 6.717 pertenciam ao sexo masculino e 6.202 ao sexo feminino, isto é, $52 \%$ de recém-nascidos masculinos para $48 \%$ de femininos, percentagens semelhantes às citadas por vários autores ${ }^{2,6,7}$. Na maternidade particular houve 3.176 recém-nascidos vivos, com uma percentagem de $50,66 \%$ de masculinos para $49,34 \%$ de femininos. Houve ai também um predominio de recém-nascidos do sexo masculino. 
SIQUEIRA, A.A.F. de et al. Relação entre peso a o nascer, sexo do recém-nascido e tipo de parto. Rev. Saúde públ., S. Paulo, 15:283-90, 1981.

Chamou a atenção o fato de que na maternidade assistencial, para crianças de peso igual ou inferior a $2.500 \mathrm{~g}$, havia $45,32 \%$ de masculinos e $54,68 \%$ de femininos, ou seja, predominância de crianças do sexo feminino. Nos demais grupos de peso, à medida que o peso aưmentou, a proporção de masculinos aumentou, e para o grupo de maiores de $4.000 \mathrm{~g}$ houve $65,60 \%$ de masculinos e apenas $34,40 \%$ de femininos (Tabela 1).

T A B E L A 1

Recém-nascidos segundo sexo e peso ao nascer, no hospital assistencial, 1974.

\begin{tabular}{|c|c|c|c|c|c|c|}
\hline \multirow[b]{2}{*}{ Peso (g) } & \multicolumn{2}{|c|}{ Masculino } & \multicolumn{2}{|c|}{ Feminino } & \multicolumn{2}{|c|}{ Total } \\
\hline & $\mathrm{No}$ & $\%$ & $\mathrm{No}$ & $\%$ & No & $\%$ \\
\hline$\leqslant 2.500$ & 760 & 45,32 & 917 & 54,68 & 1.677 & 100,0 \\
\hline $2.501-3.000$ & 2.011 & 47,06 & 2.262 & 52,94 & 4.273 & 100,0 \\
\hline $3.001-4.000$ & 3.740 & 56,20 & 2.915 & 43,80 & 6.655 & 100,0 \\
\hline$>4.000$ & 206 & 65,60 & 108 & 34,40 & 314 & 100,0 \\
\hline Total & 6.717 & 52,08 & 6.202 & 48,00 & 12.919 & 100,0 \\
\hline
\end{tabular}

Dados semelhantes foram encontrados na maternidade particular (Tábela 2).

T A B E L A 2

Recém-nascidos segindo sexo e peso ao nascer, no hospital particular, 1973.

\begin{tabular}{|c|c|c|c|c|c|c|}
\hline \multirow[b]{2}{*}{ Peso (g) } & \multicolumn{2}{|c|}{ Masculino } & \multicolumn{2}{|c|}{ Feminino } & \multicolumn{2}{|c|}{ Total } \\
\hline & No & $\%$ & Ne & $\%$ & Ne & $\%$ \\
\hline$\leqslant 2.500$ & 84 & 42,86 & 112 & 57,14 & 196 & 100,0 \\
\hline $2.501-3.000$ & 321 & 39,34 & 495 & 60,66 & 816 & 100,0 \\
\hline $3.001-4.000$ & 1.103 & 55,04 & 901 & 44,96 & 2.004 & 100,0 \\
\hline$>4.000$ & 101 & 63,12 & 59 & 36.88 & 160 & 100.0 \\
\hline Total & 1.609 & 50,66 & 1.567 & 49,34 & 3.176 & 100,0 \\
\hline
\end{tabular}

Estes dados estão de acordo com os relatados por Yerushalmy e col. ${ }^{9}$, Qunsted e col.8 e Yunes ${ }^{10}$, este em nosso meio.

Quanto ao tipo de parto, houve uma incidência de $12,37 \%$ de partos operatórios, sendo $10,08 \%$ de cesáreas, $2,29 \%$ de fórceps e $87,63 \%$ de partos normais, no hospital assistencial. Já no outro hospital houve $39,96 \%$ de cesáreas, $\mathbf{7 , 5 2 \%}$ de fórceps e $52,52 \%$ de partos normais (Tabela 3 ). 


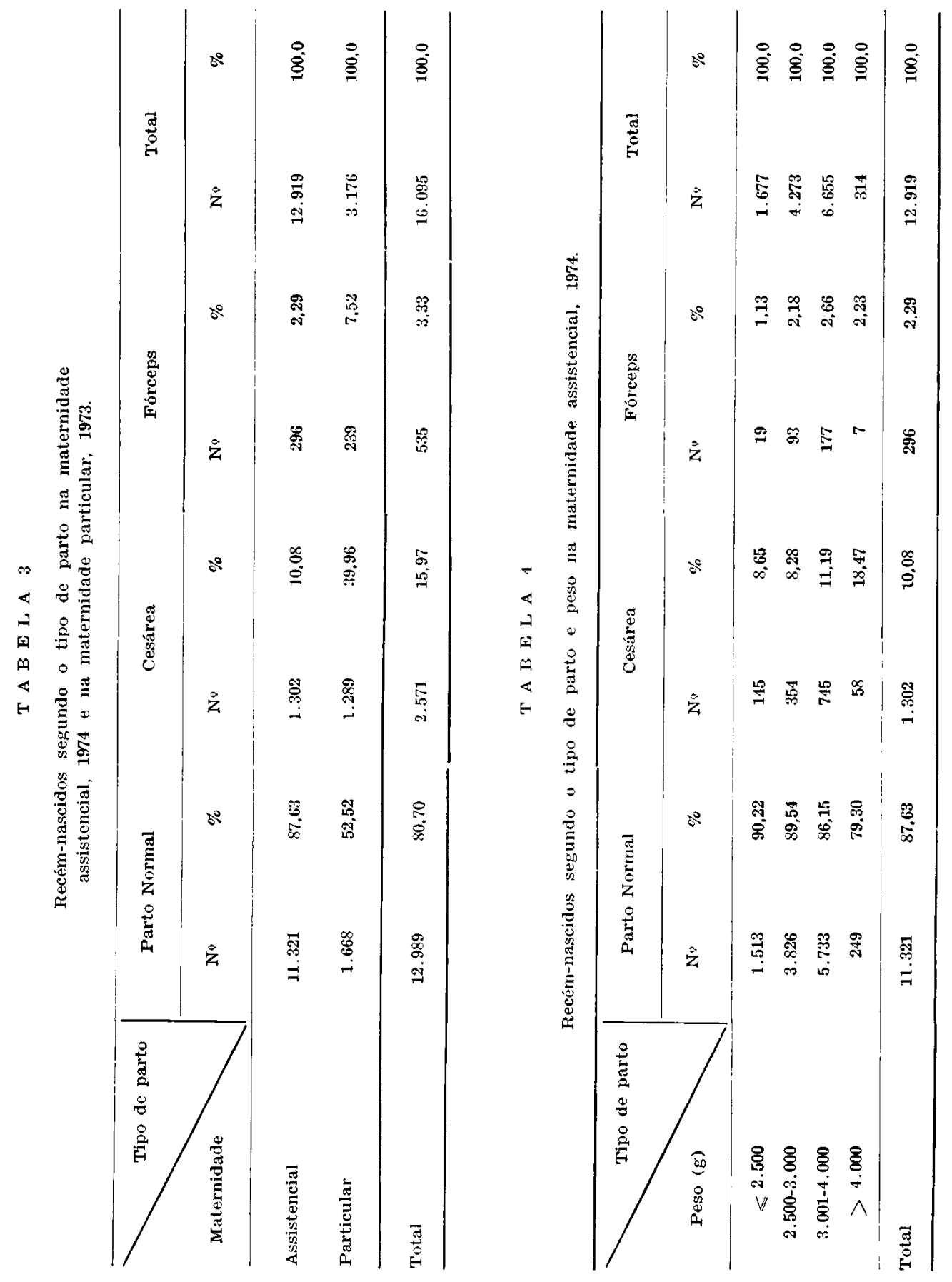



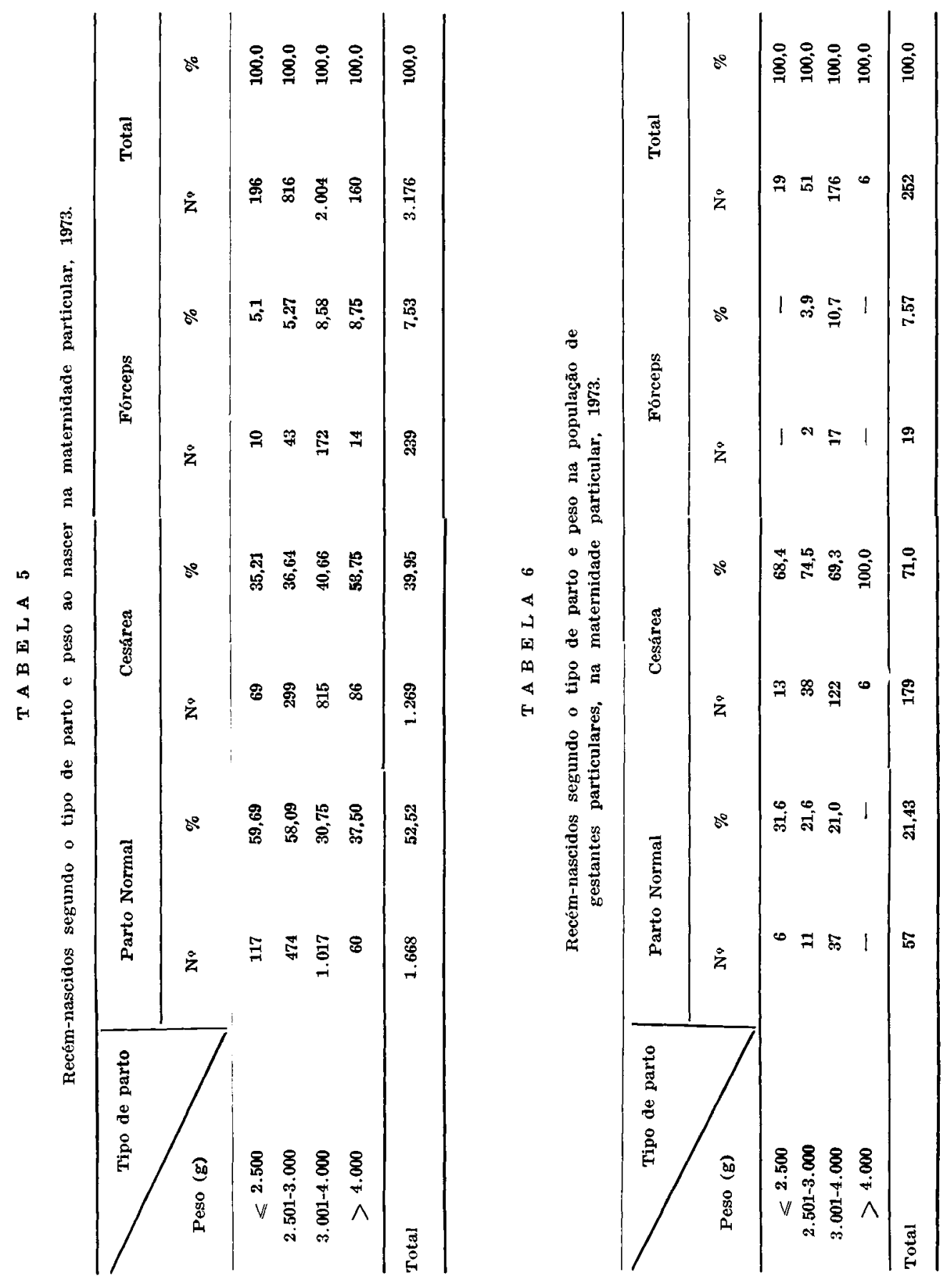
SIQUEIRA, A.A.F. de et al. Relaçāo entre peso a o nascer, sexo do recém-nascido e tipo de parto. Rev. Saúde publ., S. Paulo, 15:283-90, 1981.

T A B E L A 7

Recém-nascidos segundo o tipo de parto e peso na população previdenciária da maternidade particular, 1973.

\begin{tabular}{|c|c|c|c|c|c|c|c|c|}
\hline \multirow[b]{2}{*}{ Peso（g) } & \multicolumn{2}{|c|}{ Parto Normal } & \multicolumn{2}{|c|}{ Cesárea } & \multicolumn{2}{|c|}{ Fórceps } & \multicolumn{2}{|c|}{ Total } \\
\hline & $\mathrm{N}$ & $\%$ & $\mathrm{~N}^{\prime \prime}$ & $\%$ & $N^{\prime}$ & $\%$ & $\mathbf{N}^{\prime}$ & $\%$ \\
\hline$<2.500$ & 111 & 62.71 & 56 & 31,63 & 10 & 5,36 & 177 & 100,0 \\
\hline $2.501-3.000$ & 463 & 60.52 & 261 & 34,12 & 41 & 5,36 & 765 & 100,0 \\
\hline $3.001-4.000$ & 980 & 53.60 & 693 & 37,92 & 155 & 8,47 & 1.828 & 100,0 \\
\hline$>4.000$ & 60 & 55.19 & 80 & 51,94 & 14 & 9,10 & 154 & 100,0 \\
\hline Total & 1.614 & 55,19 & 1.090 & 37,27 & 220 & 7,54 & 2.924 & 100,0 \\
\hline
\end{tabular}

Estas diferenças entre os dois hospitais mostraram ser altamente significativas. Pode-se verificar que a incidência de partos operatórios é quase quatro vezes maior no hospital particular do que no assistencial. Considerando que no hospital assistencial a proporção de gestantes de alto risco deva ser maior do que no particular, esta diferença se torna ainda mais importante.

A Tabela 4 mostra a distribuição do tipo de parto segundo o peso do recém-nascido no hospital assistencial. $A$ incidência de cesáreas foi menor do que $9 \%$ para as crianças de $3.000 \mathrm{~g}$ ou menos, foi de $11 \%$ para as crianças de 3.001 a $4.000 \mathrm{~g}$, e alcançou quase $20 \%$ para maiorees de $4.000 \mathrm{~g}$. Como as parturientes apresentam grande homogeneidade, especialmente no que se refere ao status sócio-econômico, a incidência dupla de cesáreas no grupo de maior peso deve-se principalmente ao maior tamanho do nascituro o que provoca mais freqüentemiente distócias por desproporção materno-fetal. As diferenças ericontradas. foram altamente significativas $\left(x^{2}=68,02\right)$, a nível de $5 \%$, mostrando associação do tamanho do feto com o tipo de parto, ou seja, quanto maior o feto maior a incidência de cesáreas.

O mesmo ocorreu para o hospital particular. A Tabela 5 mostra a distribuição do tipo de parto segundo peso ao nascer naquele hospital.

Verificou-se que a incidência de cesáreas no hospital particular, apesar de ser muito maior do que no hospital assistencial. também foi tanto maior quanto maior 0 peso do recém-nascido. $O$ teste de $x^{2}$ revelou-se significativo. $\left(\chi^{2}=36,41\right.$ a nível de $5 \%$ ).

Muitos autores tệm responsabilizado o sistema de pagamento de partos do INAMPS pelo aumento da incidência de partos operatórios, entre os quais Gentile de Mello ${ }^{5}$. Em hospitais que atendem à clientela pagante, são freqüentes as incidências de cesáreas até superiorés às aqui encontradas.

Como o hospital particular estudado também atende a uma clientela pagante, procurou-se verificar o tipo de parto conforme se tratasse de clientela particular ou previdenciária. (Tabelas 6 e 7).

Verificou-se que a incidência de cesáreas nas gestantes particulares variou de $68,4 \%$ a $100 \%$, sem apresentar relação com o peso 
SIQUEIRA, A.A.F. de et al. Relação entre peso a o nascer, sexo do recém-nascido e tipo de parto. Rev. Saude puibl., S. Paulo, 15:283-90, 1981.

do recém-nascido, ou seja, encontrou-se $\chi^{2}$ não significativo $\left(\chi^{2}=6,89\right)$. Tal fato veio mais uma vez confirmar que a clientela particular pagante apresenta incidência muito elevada de cesáreas.

Na Tabela 7 foram excluidos os partos particulares do total geral de partos do hospital particular, tornando-se mais uma vez evidente a relação do tipo de parto com o peso do recém-nascido na clientela previdenciária, se bem que as percentagens de cesáreas se mantêm muito além das encontradas no hospital assistencial, confirmando então a influência do fator econômico na escolha do tipo de parto.

\section{CONCLUSOES}

A proporção de recém-nascidos do sexo feminino foi predominante na faixa de peso igual e abaixo de $2.500 \mathrm{~g}$, tanto na maternidade assistencial como na maternidade particular. A explicação para o fato merece maior investigação científica.

A proporção de recém-nascidos do sexo masculino elevou-se com o aumento de peso. tornando-se máxima no grupo acima de $4.000 \mathrm{~g}$.

Houve associação entre o tipo de parto e o peso do recém-nascido nas duas maternidades estudadas; isto quer dizer que a incidência de cesáreas elevou-se com 0 aumento do peso do nascituro.

Não houve associação entre o tipo de parto e o peso do recém-nascido na clientela não previdenciária da maternidade particular.

SIQUEIRA, A.A.F. de et al. [The relationship among infant birth weight and sex, and type of delivery]. Rev. Saúde públ., S. Paulo, 15:283-90, 1981.

ABSTRACT - In order to assess the influence of birth weight on the type of birth two maternity hospitals whose patients were of different socio-economic levels were studied. 16,095 births were analysed. It was discovered that the incidence of cesarian sections increased with the increase in birth weight in both hospitals, but that in the private hospital the incidence was four times higher than in the hospital for the poor. No relation was found, in those women who received private treatment, between type of brith and birth weight. Among those babies who weighed $2500 \mathrm{~g}$ or less at birth, there was found to be a significant predominance of girls and for those who weighed more than $4000 \mathrm{~g}$ there was, equally, a larger proportion of boys.

UNITERMS: Birth weight. Labor. Infant newborn.

\section{REFERENCIAS BIBLIOGRAFICAS}

1. ABRAMOVICZ, M. \& KASS, E.H. Pathogenesis and prognosis of prematurity. New Engl. J. Med., 275:878-85, 1966.

2. ALCANTARA, P. \& MARCONDES, F. Pediatria bdisica. 2" ed. São Paulo, Sarvier. 1968.

3. BUTTLER, N.R. \& BONHA, M.D.C. Perinatal mortality: the first report of the
1958 British Perinatal Mortality Survey. Edinburg, Livingstone, 1963.

4. COOIE, R.E. \& LEVINE, S. Bases biologicas en la practica pediatrica. Barcelona, Salvat, 1970.

5. GENTILE DE MELLO, C. Privatização e produtividade dos serviços de saúde: 
SIQUEIRA, A.A.F. de et al. Relação entre peso a nascer, sexo do recém-nascido e tipo de parto. Rev. Saúde públ., S. Paulo, 15:283-90, 1981.

pesquisa setorial sobre a incidência de cesárea. Rev. paul. Hosp., 17(10):3-11. 1969.

6. MORISON, J.E. Patologia fetal y neonatal. $3^{q}$ ed. Barcelona, Editorial Pediatrica, 1972.

7. NELSON, N.E. et al. Tratado de pediatria. $4^{\text {a }}$ ed. Barcelona, Salvat, 1960.

8. OUNSTED, M.K. et al. Clinical assessment of gestational age at birth: the effects of sex, birthweight, and weight for lenght of gestation. Early hum. Develop. 2:73-80, 1978.

9. YERUSHALMY, J. et al. Birthweight and gestation as indices of imaturity. Amer. J. Dis. Child., 109:43-57, 1965.

10. YUNES, J. Principais caracteristicas médico-sociais do recem-nascido de baixo peso. São Paulo, 1975. [Tese de Livre Docência - Faculdade de Medicina da USP].

Recebido para publicação em 05/12/1980

Aprovado para publicaçāo em 11/03/1981 